\title{
CONTRIBUCION AL CONOCIMIENTO DE LOS PHTHIRAPTERA (ANOPLURA, HOPLOPLEURA) PARASITOS DE ROEDORES EN CHILE
}

\section{CONTRIBUTION TO THE KNOWLEDGE OF PHTHIRAPTERA (ANOPLURA, HOPLOPLEURA) PARASITES ON RODENTS IN CHILE}

\author{
Daniel González-Acuña ${ }^{1}$, Dolores del C. Castro $^{2}$ \& Lucila Moreno-Salas ${ }^{1}$ \\ ${ }^{1}$ Facultad de Medicina Veterinaria, Universidad de Concepción, Casilla 537, Chillán, Chile. \\ ${ }^{2}$ Facultad de Ciencias Naturales y Museo de la Plata, Paseo del Bosque s/n, 1900, La Plata, Buenos Aires, Argentina. \\ Correspondencia autor: E-mail: danigonz@udec.cl
}

\section{RESUMEN}

Se cita por primera vez a Hoplopleura travassosi Werneck 1932 como asimismo su hospedero, Oligoryzomys longicaudatus (Bennett 1832) para Chile. Además, se amplía la distribución de Hoplopleura andina Castro 1981 sobre Akodon olivaceus Waterhouse, 1835, desde la VIII hasta la V Región de Chile. El estatus de las especies neotropicales del género Hoplopleura y su distribución geográfica actual es discutido.

Palabras claves: Hoplopleura andina, Hoplopleura travassosi, Phthiraptera, roedores, nueva cita, distribución, Chile.

\section{ABSTRACT}

This is a first report from Chile, of Hoplopleura travassosi Werneck 1932 and its host Oligoryzomys longicaudatus (Bennet 1833). New data of distribution in Chile of Hoplopleura andina Castro 1981 a parasite of Akodon olivaceus Waterhouse, 1853 is given. Status of neotropical species of genus Hoplopleura and their present geographical distribution is discussed.

Keywords: Hoplopleura andina, Hoplopleura travassosi, Phthiraptera, rodents, new record, distribution, Chile.

\section{INTRODUCCION}

Hoplopleura Enderlein, 1904 es un género cosmopolita representado en la actualidad por unas 145 especies, se ha descrito como parásito del orden Rodentia de las familias Muridae, Echymidae, Octodontidae y Siuridae, además del orden Lagomorpha de la familia Ochotonidae. En la región neotropical hasta la fecha, el mencionado género está representado por 48 especies, todas parásitas de roedores sigmodontinos (Castro 1981).
Hoplopleura andina fue descrita sobre su hospedero tipo, Akodon olivaceus de Concepción, Chile. Ampliamos aquí su distribución y citamos por primera vez a Hoplopleura travassosi y a su hospedero, Oligoryzomys longicaudatus para Chile.

\section{MATERIAL Y METODOS}

Los Phthiraptera fueron colectados por peinado exhaustivo de los hospederos y fijados en alcohol 
$70 \%$. Posteriormente, siguiendo la metodología descrita por Palma (1978), fueron limpiados en $\mathrm{KOH}$ al $20 \%$, luego se pasaron por soluciones ascendentes de alcohol $(40 \%, 80 \%$ y $100 \%)$, se aclararon durante 24 horas en aceite de clavo, para finalmente montarlos en Bálsamo de Canadá. Los ejemplares estudiados fueron depositados en la colección Zoológica del Departamento de Ciencias Pecuarias, Laboratorio de Zoología, Universidad de Concepción, Chillán, Chile.

\section{RESULTADOS}

Hoplopleura travassosi Werneck 1932 (nueva cita)

Hoplopleura torresi, Ronderos y Capri 1966.

Hospedero tipo: Oligoryzomys flavescens (Waterhouse).

Otros hospederos: Calomys callosus (Renger), $C$. laucha (Fischer), Oecomys speciosus (J. A. Allen \& Chapman), O. trinitatis (J. A. Allen \& Chapman), Oligoryzomys delticola Thomas, O. fulvescens (Saussure), O. flavescens y O. longicaudatus (Bennett).

Material examinado: $2 \circ, 10^{*}$ sobre Oligoryzomis longicaudatus, Talca, VIII Región y 1 , igual hospedero, Coyhaique, XI Región, Chile, septiembre de 2002. D. González-Acuña.

\section{Hoplopleura andina Castro 1981}

Hospedero tipo: Akodon olivaceus (Waterhouse) Otros hospederos: Akodon xanthorhinus (Waterhouse), A. olivaceus, A. longipilis (Waterhouse), Achroeomys andinus (Philippi) y Geoxus valdivianus (Philippi).

Material examinado: 1 i sobre Akodon olivaceus, Reserva Forestal Peñuelas, V Región, Chile, septiembre 2002. D. González-Acuña.

\section{DISCUSION}

Hoplopleura travassosi, hasta el presente trabajo estaba citada para distintos hospederos todos ellos Muridae: Sigmodontinae cuya distribución comprendía Venezuela, Brasil, Uruguay y Argentina (Durden \& Muser 1994, Castro y González 1997,
Castro y Cicchino 1998). Con el presente registro se amplía su distribución a Chile, además de citar a su hospedero $O$. longicaudatus por primera vez para Chile. Asimismo H. andina, en su descripción original, fue citada sobre su hospedero tipo Akodon olivaceus de Concepción, Chile y sobre A. andinus de Mendoza, Argentina (Castro 1981). Posteriormente, Castro (1982) la cita para Argentina sobre A. xanthorhinus de Ushuaia, Tierra del Fuego y Castro y Cicchino 1998 sobre A. longipilis de Ushuaia, Tierra del Fuego. Durden y Musser (1994), siguiendo el criterio de Musser y Carleton (1993) ubican a $A$. andinus en el género Chroeomys, criterio que seguimos en esta contribución. Gómez (1998) la cita asimismo sobre Geoxus valdivianus de Lonquimay, Chile. Esta especie de Hoplopleura, parásita de roedores Muridae: Sigmodontidae presenta hasta la fecha una distribución restringida a Chile y Argentina. Con el presente reporte se amplía su distribución para Chile desde la VIII y IX a la V Región.

Cabe destacar que de la nómina de 48 especies neotropicales de Hoplopleura mencionadas se excluyen a Hoplopleura chilensis (Werneck), H. disgrega (Ferris), H. griseoflavae (Castro) y $H$. octomydis (Castro et al.) debido a que, según Castro \& Verzi (2002), pertenecen al género Ferrisella Ewing 1929, género que comprende un total de 5 especies parásitas de Octodontidae, hasta la fecha restringidas a Bolivia, Chile y Argentina.

\section{AGRADECIMIENTOS}

Al señor Juan Carlos Torres-Mura por facilitar material de su colección particular de roedores para la ejecución del presente estudio.

\section{BIBLIOGRAFIA}

CAstro, D. DEl C. 1981. Contribución al conocimiento de los Anoplura neotropicales. Revista Sociedad entomológica Argentina 40 (1-4): 231-236.

CAstro, D. DEl C. 1982. Las especies del grupo travassosi del género Hoplopleura Enderlein, 1904, en la República Argentina. Revista Sociedad Entomológica Argentina 41 (1-4): 171-182

CAstro, D. Del C. \& A. GonzÁlez 1997. Las especies del género Hoplopleura Enderlein, 1904 (Phthiraptera, Insecta) parásitas de roedores de la región neotropical. Papeis Avulsos de Zoolo- 
gía 40 (13): 203-215.

Castro, D. del C. y A. Cicchino. 1998. Capítulo 10. Anoplura.:125-139. En J. J. Morrone y S. Coscarón (editores), Biodiversidad de Artrópodos de Argentina. Edic. Sur, La Plata, Buenos Aires, 1998.

Castro, D. del C. \& D. Verzi. 2002. A new species of Ferrisella (Phthiraptera, noplura, Hoplopleuridae) parasitic on the desert-adapted rodent Tympanoctomys barrerae (Rodentia, Octodontidae). Rudolstädter Nat. Hist. Schr. 4: 113-123.

Durden, L. A. \& G. G. Muser. 1994. The sucking lice (Insecta-Anoplura) of the world: A taxonomic checklist with records of mammalian host and geographical distribution. Bulletin of the American
Museum of Natural History 218, 99 pp.

Gómez, M. S. 1998. Two Anoplura species from rodents in Chile: Hoplopleura andina Castro, 1981 (Hoplopleuridae) from Geoxus valdivianus (Cricetidae) and Eulinognathus chilensis n. sp (Polyplacidae) from Abrocoma bennetti (Abrocomidae). Research and Reviews in Parasitology 58 (1): 49-54.

Musser, G. \& M. Carleton. 1993. Family Muridae. In: D. E. Wilson and D. M. Reeder (eds.) Mammals species of the word, a taxonomic and geographic reference, $2^{\text {nd }}$. ed., Washington, D. C., pp 501755. Smithsonian Inst. Press.

Palma. R. (1978): Slide-mounting of Lice: a Detailed Description of the Canada Balsam technique. The New Zealand Entomologist 6 (4), 432-436.

Fecha de recepción: 20/12/02

Fecha de aceptación: 28/03/03 